\title{
Contributors of main entries
}

Ahmad, Syed (Prof.), McMaster University, Hamilton, Ontario, Canada

Demand for Money: Keynesian Approach

Backhouse, Roger E. (Prof.), University of Birmingham, Birmingham, UK

Keynesian Cross; Say's Law

Baddeley, Michelle (Dr), Gonville and Caius College, Cambridge, UK

Investment: Accelerator Theory of; Speculative Bubbles

Bain, Andrew D. (Hon. Prof.), University of Glasgow, Glasgow, Scotland, UK

Demand for Money: Friedman's Approach

Benz, Matthias, University of Zurich, Zurich, Switzerland

Business Cycles: Political Business Cycle Approach

Blaug, Mark (Vis. Prof.), University of Amsterdam, Amsterdam, Netherlands

Endogenous Growth Theory

Bleaney, Michael (Prof.), University of Nottingham, Nottingham, UK

Purchasing Power Parity Theory

Boumans, Marcel (Dr), University of Amsterdam, Amsterdam,

Netherlands

Calibration

Burmeister, Edwin (Prof.), Duke University, Durham, NC, USA

Samuelson, Paul A. 
xvi Contributors of main entries

Colander, David C. (Prof.), Middlebury College, Middlebury, VT, USA Tobin, James

Cornwall, John L. (Prof.), Dalhousie University, Halifax, Nova Scotia, Canada

Catching Up and Convergence; Evolutionary Macroeconomics

Cornwall, Wendy (Prof.), Mount St. Vincent University, Halifax, Nova Scotia, Canada

Catching Up and Convergence; New Political Macroeconomics

Cross, Rod B. (Prof.), University of Strathclyde, Glasgow, Scotland, UK Hysteresis

Davidson, Paul (Prof.), University of Tennessee, Knoxville, TN, USA Post Keynesian Economics

Dawson, Graham J.A. (Dr), Open University, Milton Keynes, UK Ecological Macroeconomics; Inflation: Costs of; Inflation: Costs of Reducing; Menu Costs

Demirbas, Dilek (Dr), University of Northumbria, Newcastle-upon-Tyne, UK

Optimum Currency Area

De Vanssay, Xavier (Assoc. Prof.), York University, Toronto, Ontario, Canada

Marshall-Lerner Condition

De Vroey, Michel (Dr), Université Catholique de Louvain, Louvain-LaNeuve, Belgium

Involuntary Unemployment in Keynes's General Theory; Involuntary Unemployment in Keynesian Economics; Keynesian Economics: Reappraisals of 
Dimand, Robert W. (Prof.), Brock University, St. Catharine's, Ontario, Canada.

Balance of Payments: Keynesian Approach; Hicks, John R.; Real Balance Effect; Ricardian Equivalence; Schools of Thought in Macroeconomics

Dixon, Huw D. (Prof.), University of York, York, UK

Real Rigidity

Dore, Mohammed H.I. (Prof.), Brock University, St. Catharine's, Ontario, Canada

Representative Agent Model

Dow, Sheila C. (Prof.), University of Stirling, Stirling, Scotland, UK

Money Supply: Endogenous or Exogenous?

Dowd, Kevin (Prof.), University of Nottingham Business School,

Nottingham, UK

Gold Standard; Time Inconsistency

Eijffinger, Sylvester C.W. (Prof.), Tilburg University, Tilburg, Netherlands.

Central Bank Accountability and Transparency; Central Bank Independence

Falvey, Rod (Prof.), University of Nottingham, Nottingham, UK

Comparative Advantage

Fender, John (Prof.), University of Birmingham, Birmingham, UK

Nominal Rigidity

Fletcher, Gordon (Dr), University of Liverpool, Liverpool, UK

Neoclassical Synthesis

Frazer, William (Prof.), University of Florida, Florida, Gainesville, FL, USA

Friedman, Milton 
xviii Contributors of main entries

Frey, Bruno S. (Prof.), University of Zurich, Zurich, Switzerland Business Cycles: Political Business Cycle Approach

Garrison, Roger W. (Prof.), Auburn University, Auburn, AL,USA

Business Cycles: Austrian Approach

Gausden, Robert (formerly at the University of Northumbria, Newcastleupon-Tyne, UK)

Absolute Income Hypothesis; Permanent Income Hypothesis

Gerrard, Bill (Dr), University of Leeds, Leeds, UK

Keynes's General Theory

Grieve Smith, John, Robinson College, Cambridge, UK

Bretton Woods

Hammond, J. Daniel (Prof.), Wake Forest University, Winston-Salem, NC, USA

Business Cycles: Monetarist Approach

Hamouda, Omar F. (Prof.), York University, North York, Ontario,

Canada

Keynes, John Maynard

Hargreaves Heap, Shaun P. (Dr), University of East Anglia, Norwich, UK

New Keynesian Economics

Harrington, Richard L., University of Manchester, Manchester, UK Classical Dichotomy

Healey, Nigel M. (Prof.), Manchester Metropolitan University, Manchester, UK

AD-AS Model; Credibility and Reputation 
Holden, Ken (Prof.), Liverpool John Moores University, Liverpool, UK Forecasting; Macroeconometric Models; Vector Autoregressions

Howitt, Peter (Prof.), Brown University, Providence, RI, USA Coordination Failures

Humphrey, Thomas M. (Vice President and Economist), Federal Reserve Bank of Richmond, Richmond, VA, USA

Adaptive Expectations; Balance of Payments: Monetary Approach

Hunt, Andrew, University of Northumbria, Newcastle-upon-Tyne, UK Incomes Policy

Jackson, Peter M. (Prof.), University of Leicester, Leicester, UK

Budget Deficits: Cyclical and Structural

Junankar, P.N. Raja (Prof.), University of Western Sydney, Macarthur, Campbelltown, NSW, Australia

Investment: Neoclassical Theories of

Laidler, David E.W. (Prof.), University of Western Ontario, London, Ontario, Canada

Quantity Theory of Money

Leeson, Robert (Assoc. Prof.), Murdoch University, Perth, Australia Expectations-augmented Phillips Curve

Mayer, Thomas (Prof.), University of California, Davis, CA, USA

Monetarism; Monetary Policy: Role of

McCombie, John (Dr), Downing College, Cambridge, UK

Balance of Payments-constrained Economic Growth

Middleton, Roger (Dr), University of Bristol, Bristol, UK

Solow, Robert $M$. 
Minford, A. Patrick L. (Prof.), University of Wales, Cardiff, Wales, UK Supply-side Economics

Mishkin, Frederic S. (Prof.), Columbia University, New York, NY, USA Inflation Targeting

Mizen, Paul (Dr), University of Nottingham, Nottingham, UK

Credit Channels; Demand for Money: Buffer Stocks

Mulhearn, Chris J. (Dr), Liverpool John Moores University, Liverpool, UK

Expenditure Reducing Policy; Expenditure Switching Policy

O’Brien, Denis P. (Prof.), University of Durham, Durham, UK

Classical Economics

Peston, Maurice (Prof.), University of London, London, UK

Crowding Out; IS-LM Model: Closed Economy; IS-LM Model: Open Economy

Reuten, Geert (Dr), University of Amsterdam, Amsterdam, Netherlands

Business Cycles: Marxian Approach; Marxian Macroeconomics: An Overview; Marxian Macroeconomics: Some Key Relationships

Ryan, Cillian (Dr), University of Birmingham, Birmingham, UK Business Cycles: Real Business Cycle Approach; Business Cycles: Stylized Facts

Sandilands, Roger J., University of Strathclyde, Glasgow, Scotland, UK Great Depression

Setterfield, Mark (Assoc. Prof.), Trinity College, Hartford, CT, USA Inflation: Alternative Theories of

Shaw, G.K. (Prof.), University of Buckingham, Buckingham, UK Balanced Budget Multiplier; Keynesian Economics 
Sheffrin, Steven M. (Prof.), University of California, Davis, CA, USA Fiscal Policy: Role of

Shone, Ronald, University of Stirling, Stirling, Scotland, UK

Exchange Rate Determination: Monetary Approach

Simkins, Scott P. (Assoc. Prof.), North Carolina A and T State University, Greenboro, NC, USA

Lucas Critique; Theory and Measurement in Macroeconomics: Role of

Smithin, John (Prof.), York University, North York, Ontario, Canada Phillips Curve

Snowdon, Brian, University of Northumbria, Newcastle-upon-Tyne, UK Business Cycles: New Classical Approach; Economic Growth and the Role of Institutions; Growth Accounting; Harrod-Domar Growth Model; Lucas, Robert E. Jr.; Modigliani, Franco; Multiplier; Mundell, Robert A.; New Classical Economics; Rules versus Discretion

Solow, Robert M. (Prof.), Massachusetts Institute of Technology, Cambridge, MA, USA

Neoclassical Growth Model

Spindler, Zane A. (Prof.), Simon Fraser University, Burnably, British Columbia, Canada

Laffer Curve

Stevenson, Andrew (Honorary Senior Research Fellow), University of Glasgow, Glasgow, Scotland, UK

Fixed Exchange Rate System; Flexible Exchange Rate System

Thompson, John L. (Prof.), Liverpool John Moores University, Liverpool, UK

Natural Rate of Unemployment; Rational Expectations 
xxii Contributors of main entries

Trautwein, Hans-Michael (Prof.), Carl von Ossietzky University

Oldenburg, Germany

Credit Views in Macroeconomic Theory

Trigg, Andrew B., Open University, Milton Keynes, UK

Business Cycles: Keynesian Approach

Vane, Howard R. (Prof.), Liverpool John Moores University, Liverpool, UK

Business Cycles: New Classical Approach; Economic Growth and the Role of Institutions; Growth Accounting; Harrod-Domar Growth Model; Lucas, Robert E. Jr.; Modigliani, Franco; Multiplier; Mundell, Robert A.; New Classical Economics; Rules versus Discretion

Visser, Hans (Prof.), Free University of Amsterdam, Amsterdam, Netherlands

Neutrality of Money

Went, Robert (Dr), University of Amsterdam, Amsterdam, Netherlands Globalization

Wray, L. Randall (Prof.), University of Missouri, Kansas City, MO, USA Financial Instability 